\title{
JOURNAL.RU
}

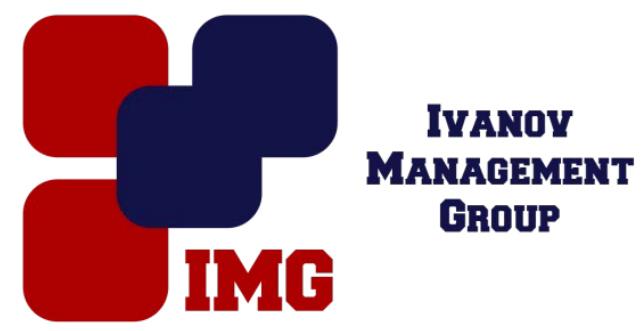

Лифинцев М. А., Мандрыкина Н. Е., Кичигина А. К., Свиридова И. В. Белгородский государственный наииональный исследовательский университет Белгород, Россия

doi: 10.18411/lj-31-01-2017-1-05

idsp 000001:lj-31-01-2017-1-05

\section{Об автоматизации программного сравнения методов СЛАУ}

Решение систем линейных алгебраических уравнений (СЛАУ) - одна из основных задач вычислительной линейной алгебры. Значительная часть численных методов решения различных задач включает в себя решение систем линейных уравнений как элементарный шаг соответствующего алгоритма.

В практических задачах часто бывает нужно найти решение, удовлетворяющее большому числу возможно противоречивых требований. Такая задача сводится к системе линейных уравнений

$$
\left\{\begin{array}{rl}
a_{11} x_{1}+a_{12} x_{2}+\ldots+a_{1 n} x_{n} & =b_{1} \\
a_{21} x_{1}+a_{22} x_{2}+\ldots+a_{2 n} x_{n} & =b_{2} \\
\ldots & \ldots \\
a_{m 1} x_{1}+a_{m 2} x_{2}+\ldots+a_{m n} x_{n} & =b_{m}
\end{array} \Longleftrightarrow A X=\mathcal{B}\right.
$$

при числе уравнений $\mathrm{m}$ большем числа неизвестных $\mathrm{n}$, то такая переопределенная система, как правило, несовместна. В этом случае задача может быть решена только путем выбора некоторого компромисса - все требования могут быть удовлетворены не полностью, а лишь до некоторой степени [2]. Псевдорешение системы $\mathrm{AX}=\mathrm{B}$ называется столбец $X \in \mathbb{R}^{n}$, обеспечивающий минимум величины

$$
\sum_{i=1}^{m}\left[a_{i 1} x_{1}+a_{i 2} x_{2}+\ldots+a_{i n} x_{n}-b_{i}\right]^{2} .
$$

Такому определению можно также соотнести вероятностную интерпретацию. Пусть для определения неизвестных величин $x_{1} \ldots x_{n}$ проводятся $\mathrm{m}$ экспериментов, описываемых линейными уравнениями:

$$
a_{i 1} x_{1}+a_{i 2} x_{2}+\ldots+a_{i n} x_{n}=b_{i} \quad \text { npu } \quad i \in\{1, \ldots, m\} .
$$

При этом величины $\left\{a_{i j}\right\}, i \in\{1, \ldots, m\}, j \in\{1, \ldots, n\}$ - известные постоянные, не подверженные сопутствующим экспериментам погрешностям, а 
вот величины $\left\{b_{i}\right\}_{i=1}^{m}$ этим погрешностям подвержены. Формально каждое из равенств следует рассматривать как приближенное. Понятно, что при таких обстоятельствах не имеет смысла гоняться за точным решением системы $\mathrm{AX}=\mathrm{B}$. Искать следует приближенное решение, оптимальное в некотором смысле.

Существует псевдорешение системы $\mathrm{AX}=\mathrm{B}$ и оно является решением системы $\left[\mathrm{A}^{\mathrm{T}} \mathrm{A}\right]=\mathrm{A}^{\mathrm{T}} \mathrm{B}$. Это решение будет единственным тогда и только тогда, когда $\operatorname{rank} \mathrm{A}=\mathrm{n}$.

Система $\left[\mathrm{A}^{\mathrm{T}} \mathrm{A}\right]=\mathrm{A}^{\mathrm{T}} \mathrm{B}$ называется нормальной системой по отношению к системе $\mathrm{AX}=\mathrm{B}$. Формально она получается домножением системы $\mathrm{AX}=\mathrm{B}$ слева на матрицу $\mathrm{A}^{\mathrm{T}}$. Заметим также, что если $\mathrm{m}=\mathrm{n}$ и $\operatorname{del} \mathrm{A} \neq 0$, то всевдорешение системы совпадает с решением в традиционном смысле.

Если нормальная система имеет бесконечное количество решений, то обычно в качестве псевдорешения берут какое-то одно из них - как правило, у которого минимальна сумма квадратов компонент («длина»).

В качестве языка программирования для автоматизации программного сравнения СЛАУ был выбран язык С++. Это очень мощный язык, позволяющий создавать программы любого назначения и любой сложности[1].

Определившись с выбором подходящего компилятора, можно приступать к написанию самой программы. В начале работы с программой высвечивается форма, которая требует ввода размерности матрицы, а затем введения элементов.

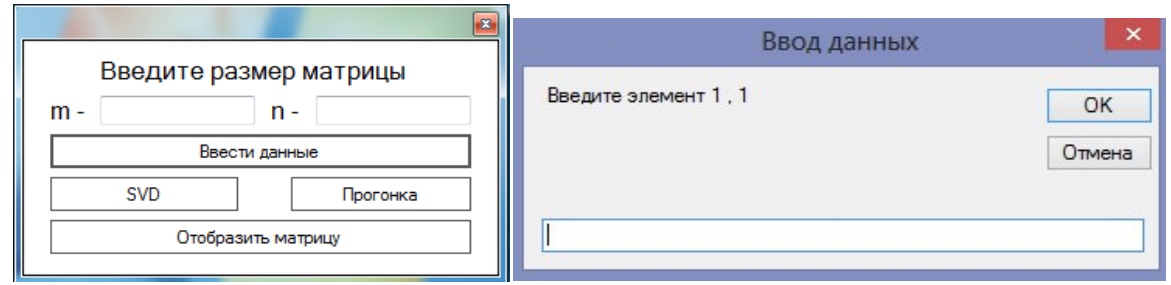

Рисунок 3, 4-Формы требующие ввести размерность матрицы и ее элементы

После ввода всех необходимых данных в программу, проводится решение систем методом SVD и методом прогонки, которые выбираются пользователем самостоятельно.

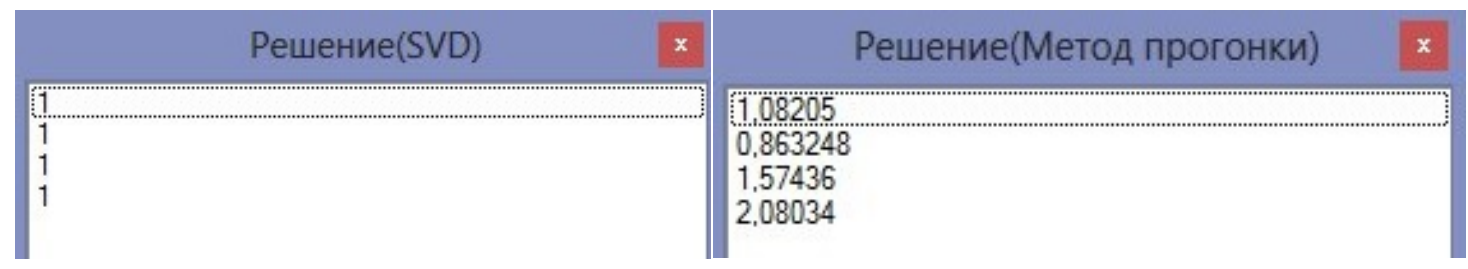

Рисунок 5, 6-Решение методом SVD и методом прогонки

Время расчета метода прогонки - 1,7секунд. Время расчета метода SVD 1,5 секунд.

Метод наименьших квадратов (SVD) дает точное решение системы алгебраических уравнений, но требует перед своим применением дополнительные преобразования - проверку на то, что количество строк больше количества столбцов. 
Во-первых, количество строк сильно зависит от матрицы А исходной системы уравнений вида $\mathrm{Ax}=\mathrm{b}$. Чем больше количество строк в уравнении, тем больше времени требуется программе, чтобы найти корни данной системы.Вовторых, на количество шагов влияет начальное приближение. Чем оно ближе к точному решению, тем меньше требуется шагов для сходимости метода.

В итоге можно сделать вывод: метод наименьших квадратов (SVD) является лучшим при решении системы линейных уравнений, он является менее объемным и требует меньше времени для нахождения корней, чем метод прогонки.

\section{Литература}

1. М. Шлее. Qt 4.8. Профессиональное программирование на С++. - СПб.: БХВ-Петербург, 2012. - с. 452

2. Численное решение переопределенных СЛАУ. Метод наименьших квадратов. [Электронный ресурс] - Режим доступа http://www.intuit.ru/studies/courses/1012/168/lecture/4594 